\title{
A Domain Wall Theory for Ferroelectric Hysteresis
}

\author{
Ralph C. Smith \\ Center for Research in Scientific Computation \\ Department of Mathematics \\ North Carolina State University \\ Raleigh, NC 27695-8205 \\ rsmith@eos.ncsu.edu \\ Craig L. Hom \\ Advanced Technology Center \\ Lockheed Martin Missiles \& Space \\ Palo Alto, CA 94304 \\ craig.hom@lmco.com
}

\begin{abstract}
This paper addresses the modeling of hysteresis in ferroelectric materials through consideration of domain wall bending and translation. The development is considered in two steps. In the first step, dielectric constitutive relations are obtained through consideration of Langevin, Ising spin and preferred orientation theory with domain interactions incorporated through mean field relations. This yields a model for the anhysteretic polarization that occurs in the absence of domain wall pinning. In the second step, hysteresis is incorporated through the consideration of domain wall dynamics and the quantification of energy losses due to inherent inclusions or pinning sites within the material. This yields a model analogous to that developed by Jiles and Atherton for ferromagnetic materials. The viability of the model is illustrated through comparison with experimental data from a PMN-PT-BT actuator operating at a temperature within the ferroelectric regime.
\end{abstract}




\section{Introduction}

An inherent property of ferroelectric materials is the presence of hysteresis in the relation between an applied field and the resulting polarization. This phenomenon is associated with the domain structure of the materials and must be accommodated in applications which employ ferroelectric components. In certain materials, such as relaxor ferroelectrics, hysteresis can be minimized in a diffuse transition region through the choice of stoichiometry and operating conditions. For example, a significant advantage of PMN-PT actuators lies in the fact that they produce large strains with minimal hysteresis when employed near their Curie point. Similarly the hysteresis exhibited by PZT actuators at low to moderate drive levels is sufficiently small to permit the use of linear design and control methodologies. However, at the high drive levels and general temperatures encountered in many current applications, the level of hysteresis in both PMN-PT and PZT elements is significant and must be accommodated to attain the full potential of these materials in high performance actuator design. At a fundamental level, the presence and nature of hysteresis loops represents a basic property characterizing ferroelectric materials. At the system level, hysteresis generates internal heat in the actuator which can cause thermal runaway in insulated systems. Highly hysteretic devices also require closed loop control for precision displacement actuation. To better understand and utilize these actuator materials, the fundamental mechanisms underlying the hysteresis must be ascertained and eventually modeled.

In an effort to provide a model which incorporates aspects of the underlying physical mechanisms and is appropriate for high performance actuator and control design, we consider a theory for ferroelectric hysteresis which is based on domain wall dynamics and energy losses due to inherent inclusions in the material. Although various types of hysteresis curves can be generated in ferroelectric materials, sigmoid curves such as that shown in Figure 1 are common in applications, and the form of hysteresis that we shall address here. This theory is the ferroelectric analogue of that developed by Jiles and Atherton for ferromagnetic materials [27].

\section{Ferroelectric Domain Theory}

It has long been recognized that domain and domain wall mechanisms within ferroelectric materials result in phenomena such as hysteresis, aging and fatigue. Initial investigations focussed primarily on mechanisms leading to domain formation, nucleation and growth in single crystal $\mathrm{BaTiO}_{3}$. Caspari and Merz provided an early analysis of the mechanisms leading to linear and quadratic strains in single crystal ferroelectric crystals [5]. They concluded that the spontaneous polarization and domain effects on the electromechanical response of the material are significant and demonstrated that a linear piezoeffect results when domain effects were minimized through the application of an external field which aligned domains. Caspari and Merz postulated that hysteresis is due to the presence of parallel and antiparallel domains which switch at various field strengths.

Fundamental investigations regarding the nucleation and growth of domains in $\mathrm{BaTiO}_{3}$ were provided by Merz [34] and Little [32], and were extended by Drougard [11] and Miller and Weinreich $[35,37]$. In concert, these investigations established that both nucleation and domain wall motion contributed to polarization reversal in $\mathrm{BaTiO}_{3}$. The dependence of polarization reversal on domain wall motion illustrated that in certain aspects, this effect is analogous to magnetization reversal in ferromagnetic materials. Subsequent experiments have elucidated further details regarding domain nucleation and growth (e.g., see [40,41]) and numerous models have been proposed to quantify domain phenomena and their effect on hysteresis and fatigue [20, 23, 24, 43, 45].

An important component in domain and domain wall theories is the effect due to impurities or inclusions in the materials. Miller and Savage observed that domain wall motion is dependent upon 
the impurity levels of the crystal and considered a model based on a surface layer adjacent to the domain wall [36]. Laikhtman extended this idea to develop a model for the bending of domain walls pinned by inclusions or defects in the material [30]. This model was developed under the premise that the application of an external field produces a force which acts as a pressure on the wall and causes it to bend in a manner analogous to a pinned membrane. The resulting model was derived using elasticity theory and accounts for the inertia of the domain wall as well as certain anisotropy and temperature effects. Finally, Laikhtman observed from experimental evidence in $[14,19]$ that domain walls exhibit an "effective viscosity" which depends on the concentration of defects. This provides an irreversible component to the polarization which Laikhtman incorporated through the inclusion of factors modeling the dissipation of heat. Finally, it is noted that this theory breaks down if the modeled wall comes in contact with remote defects. We note that an overview of the "friction" effects and dielectric losses due to domain wall motion can be found in [6] and [50, pp. 402-427].

The ideas underlying the influence of pinning sites on domain wall dynamics were extended by Fedosov and Sidorkin [12]. They concluded that for low displacement levels (on the order of the lattice constant), domain walls can be considered as moving as a whole. For higher displacement levels, however, pinning effects become significant and local bending between pinning sites should be considered. A comprehensive analysis of field, frequency and thermal effects on domain wall dynamics in relaxor ferroelectrics has been provided by Chen and Wang [7]. These authors note that at low field levels with high frequencies, the dielectric behavior of the material can be primarily attributed to the bending of domain walls pinned at inclusions. For large applied or internal fields, the local energy barriers associated with pinning sites are overcome and the domain walls move for extended distances. We note that these localized energy barriers provide a thermodynamic mechanism for describing the "effective viscosity" and "frictional" domain wall effects reported in $[30,50]$.

The combination of the bending mechanisms described in $[7,12,30]$, and the energy barriers detailed in [7] indicates that both reversible and irreversible mechanisms contribute to ferroelectric hysteresis. Furthermore, the analysis and experiments reported in these investigations indicate that these mechanisms are due to the pinning of domain walls at defects in the material and the energy input required to move domain walls across pinning sites. The discussion in the subsequent section summarizes certain previous approaches used to quantify these effects and provides a background for the model presented here.

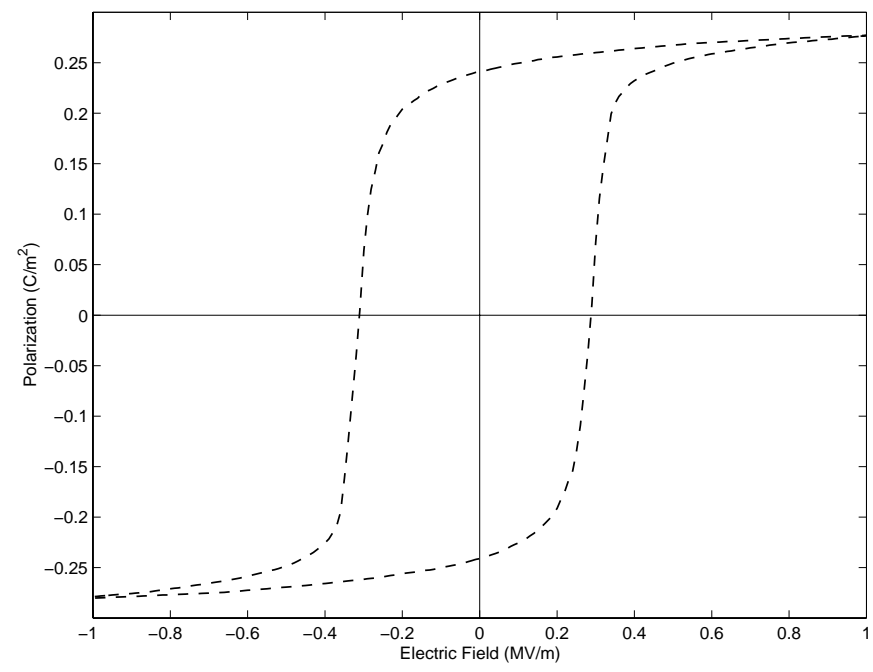

Figure 1. Hysteresis loop measured at a temperature of $T=-20^{\circ} C$ in a PMN-PT-BT sample whose Curie temperature is $T_{c}=45^{\circ} \mathrm{C}$. 


\section{Hysteresis Models}

The nature of current hysteresis models for ferroelectric materials can be roughly categorized as follows: (1) microscopic theories based on quantum mechanics, classical elasticity and electromagnetic relations, or thermodynamic laws, (2) macroscopic theories based upon phenomenological principles, or (3) semi-macroscopic theories which are derived using a combination of approaches.

Microscopic theories for ferroelectric materials generally address the previously described polarization processes at the lattice or domain level. Model development at the lattice level is illustrated by the theory of Omura et al [42]. In their approach, the free energy for a one-dimensional ferroelectric lattice system is represented in terms of the summed moments and coupling coefficients at the atoms in the lattice. Hysteresis is incorporated through the inclusion of viscosity coefficients at each lattice site. Simulation results demonstrate that the model yields reasonable families of hysteresis curves but requires a large number of degrees of freedom to accommodate the atoms in the one-dimensional lattice (the examples were generated with $N=250$ ).

For simple stoichiometries, microscopic models of this type are theoretically plausible. For the crystalline conditions encountered in present actuator designs, however, it is difficult to construct tractable microscopic models which accommodate attributes such as grain boundaries and intergranular stresses as well as the polycrystalline nature of the materials. Moreover, microscopic models at the atomic or lattice level are often difficult to employ in control design due to the large number of required states and parameters.

The domain wall models of Fedosov and Sidorkin [12] and Laikhtman [30] are also local in nature and hence would require either macroscopic averaging or a large number of parameters when employed in actuator characterization or design. However, the concepts underlying these models are in accordance with the approach employed in this paper for modeling the reversible component of the polarization with the primary difference in philosophy residing in our use of macroscopic parameters which quantify "average" properties of the material.

Numerous macroscopic models for hysteresis processes in ferroelectric materials have been derived using empirical or phenomenological principles. In some cases, these models are motivated by physical domain processes while other models circumvent poorly understood physics through purely mathematical characterizations. Models in the latter category are advantageous when the underlying physics is difficult to quantify. Due to their nature, however, it is difficult to employ known physics or physical measurements to directly construct such purely phenomenological models or update them to accommodate changing operating conditions.

A large class of hysteresis models have been derived through fits obtained with hyperbolic tangent and cotangent functions. As detailed in Section 2 of this paper, Langevin functions involving the hyperbolic cotangent and Ising spin models yielding the hyperbolic tangent arise when Boltzmann statistics are used to derive the anhysteretic (hysteresis-free) response in ferroelectric and ferromagnetic materials. These representations provide natural envelops for phenomenological quantification of hysteresis families.

Hom and Shankar have demonstrated that certain hysteresis curves can be generated through the appropriate choice of parameters in the Ising spin model [22]. As illustrated by their results, however, this method produces hysteresis curves in which the transition from one saturation region to the other is much steeper than that observed in typical ferroelectric applications.

More gradual transitions can be obtained by employing Langevin or hyperbolic cotangent functions which are shifted to coincide with the remanence polarizations. This technique was employed by Zhang and Rogers when modeling hysteresis in piezoceramic materials [54] and Miller et al for characterization hysteresis in ferroelectric capacitors [38]. While this technique often provides accurate model fits with a small number of required parameters (in some cases two), its capabilities 
for general applications involving symmetric and asymmetric minor loops appears limited. In the context of the underlying physics, the shifting of the anhysteretic curves provides a phenomenological mechanism for modeling the "viscosity" or energy losses which occur during domain growth through domain wall movement.

Another class of phenomenological hysteresis models are based upon the determination of empirical laws which quantify the rate of dipole or polarization switching. Based on observations by Merz [34], Landauer et al [31] proposed a model which quantifies the rate of polarization switching in terms of the current and saturation polarization and applied field. Applying a similar philosophy, Chen and Montgomery [6] developed a model in which the rate at which dipoles align with the applied field is specified as a nonlinear function of the field. Both approaches provide phenomenological characterizations of the rate at which domain walls move.

Preisach and generalized Preisach models have also been widely used to characterize hysteresis in ferroelectric materials. In their classical form, Preisach operators are constructed from linear combinations of multivalued kernels [33]. The coefficients for each kernel represent the input level and magnitude when switching occurs between two saturation states which define the kernel. When used to quantify magnetic hysteresis, the kernel has been interpreted as representing diametrically opposite dipole configurations with the coefficients interpreted as quantifying the field inputs required for dipole switching and degree to which they switch at the points. While a similar interpretation can be made for ferroelectric processes, the techniques are more commonly considered as phenomenological and hence provide a purely mathematical model for the process. Generalized Preisach or Krasnoselskii-Pokrovskii models differ through the choice of kernel. As detailed in [2], the classical Preisach operators are discontinuous with respect to both the parameters and time. This is alleviated in Krasnoselskii-Pokrovskii operators through the use of smooth ridge functions which provide an envelop of admissible families. We note that the construction of Krasnoselskii-Pokrovskii kernels through translations of ridge functions is quite similar in philosophy to the use of shifted anhysteretic curves employed in the models of Miller et al [38] and Zhang and Rogers [54].

The modifications necessary for employing the classical Preisach techniques for ferroelectric hysteresis are illustrated by the models developed by Ge and Jouaneh $[17,18]$. As illustrated by both numerical and experimental examples, this technique has been used accurately characterize both major and minor hysteresis loops in piezoceramic actuators. The development of Krasnoselskii-Pokrovskii hysteresis models for piezoceramic actuators has been considered by Galinaitis and Rogers [15, 16]. References [16] also provides an overview concerning the construction of inverse operators for control methods based on output linearization.

Finally, semi-macroscopic models are typically derived using a combination of thermodynamic and phenomenological principles. The goal in these approaches is to employ energy-based relations to the extent possible so that known physics can be used to facilitate model construction and updating. Phenomenological principles contribute to aspects of the constitutive equations and the development of macroscopic averages of microscopic properties such as the intergranular stresses and domain wall pinning site energies.

An illustration of this type of approach can be found in the hysteresis model of Yoo and Desu for ferroelectric thin films [53]. The hysteresis is characterized through consideration of three stages in the polarization process: domain nucleation and growth, domain merging, and domain shrinkage. The dynamics in these stages are developed through the combination of physical mechanisms underlying polarization reversal and macroscopic averages of certain domain properties. The domain wall model of Chen and Wang [7] for relaxor ferroelectric materials can also be considered as semi-macroscopic. In their development, Chen and Wang employ energy relations in combination with phenomenological relations to obtain microscopic polarization rate equations and corresponding macroscopic constitutive equations. Aspects of the model are formulated in the framework of 
dislocation theory for plasticity and anelasticity which leads to a fairly general model for domain wall dynamics. While low temperature hysteresis loops are used to provide evidence of domain wall motion, this investigation does not address per se the modeling of hysteresis in relaxor ferroelectric materials.

This paper describes a hysteresis model which is semi-macroscopic in nature and is based upon the quantification of the domain wall energy in the material. For an ideal material which is free from inclusions or second-phase materials, domain wall movement is unimpeded and the relation between applied stresses, electric fields and temperatures, and the resulting hysteresis-free or anhysteretic polarization is modeled by nonlinear constitutive equations. As detailed in [22], appropriate constitutive relations are derived through thermodynamic theory combined with phenomenological relations such as Hooke's law. In actual materials, however, domain wall movement is impeded by inherent inclusions within the material. The model presented here quantifies the resulting hysteresis through consideration of the energy required to translate domain walls and break pinning sites. An 'average' measure of this energy is employed to obtain a model which requires the identification of only five parameters. Due to the small number and physical nature of certain parameters (e.g., the saturation polarization $P_{s}$ ), the model is easily constructed and updated. We note that this model is the ferroelectric analogue of that developed by Jiles and Atherton for ferromagnetic materials [27]. While aspects of the Jiles/Atherton theory were employed in [51] to characterize hysteresis in stress/strain relations for $\mathrm{LiCsSO}_{4}$ crystals, this is the first application of this approach to quantify hysteresis in the $E-P$ relation for ferroelectric materials. We also point out that while certain ferroelectric actuator materials provide the motivation, the model is general in nature and can be applied to a variety of ferroelectric materials including capacitor and electro-optic materials.

Certain aspects of the constitutive theory of Hom and Shankar are summarized in Section 2. This discussion includes the development of the classical Langevin and Ising spin models as well as a third model which combines attributes of the two. This section also provides a discussion concerning the incorporation of interdomain coupling to provide the effective field observed at the domain level and outlines the extension of the models from the microscopic to macroscopic levels. The domain wall theory contributing to both reversible and irreversible changes in polarization is presented in Section 3. To simplify the development, the final hysteresis model is formulated through scalar-valued relations between the applied field $E$ and resulting polarization $P$. This formulation is suitable for a variety of current applications and materials. At various points in the development, however, intermediate vector-valued relations are required and the notation $\mathbf{E}$ and $\mathbf{P}$ are used to denote the respective field and polarization in $\mathbb{R}^{3}$. This also provides a framework for developing the corresponding vector-valued hysteresis model if required by the application. In the final section, the viability of the model is illustrated through comparison with experimental data from a PMN-PT-BT actuator.

\section{Constitutive Theory for Ferroelectric Materials}

In this section we consider the development of constitutive equations which describe the anhysteretic (hysteresis-free) mechanical and dielectric behavior of certain ferroelectric materials. Hysteresis effects are included in the next section through quantification of energy loss due to pinning sites in the material.

Following classical developments for electrostrictive materials, the constitutive relations are assumed to be of the form

$$
\begin{aligned}
& \sigma=Y\left(e-Q_{33} P^{2}\right) \\
& E=-2 Q_{33} P \sigma+\mathcal{F}(T, P) .
\end{aligned}
$$


Here $\sigma$ denotes the axial stress, $e$ denotes the longitudinal strain, $Y$ is the closed-circuit elastic modulus, $T$ is the temperature, $P$ denotes the polarization and $Q_{33}$ denotes the longitudinal electrostrictive coupling coefficient. We note that these constitutive relations can be modified for piezoelectric materials by replacing the quadratic polarization term in the mechanical relation by a linear term.

In the remainder of the section, we summarize three techniques for modeling the function $\mathcal{F}$ which quantifies the dependence of the electric field $E$ on the temperature and polarization. All three models are based upon the application of Boltzmann's law to various dipole orientations within the ferroelectric crystal. This follows the approach employed by Hom and Shankar [21, 22] and in one case provides a Langevin model analogous to that typically employed in ferromagnetic applications. We note that other techniques can be employed for modeling the anhysteretic constitutive relations for certain ferroelectric materials and refer the reader to $[8,44]$ for discussion concerning two alternative models.

\subsection{Noninteracting Cells}

We view a ferroelectric crystal as a lattice of cells, where each cell possesses a permanent dipole moment with an associated direction. Normally, the cell has a finite number of possible orientations (eight for a tetragonal system). When free of electric field and above the Curie temperature, thermal agitation creates a random distribution of orientations and the ceramic has an average polarization of zero. The application of an electric field forces some dipole moments to switch to an orientation closer to the direction of the field. The resulting distribution is then no longer random and a macroscopic polarization develops. We point out that collections of neighboring cells having the same polarization form the domain structure of the crystal. The degree of switching increases with increasing field until the global polarization eventually saturates when all the cells have optimally oriented their dipole moments relative to the field. For the development in this section, we will assume that the cells do not interact and use statistical mechanics to derive three nonlinear models for the dielectric response of this system. The models differ only in the assumptions made about the cells' possible orientation. The effects of cell interaction are then considered in Section 2.2.

For a dipole moment $\mathbf{p}_{0}$ in an electric field $\mathbf{E}$, the potential energy is given by

$$
\mathcal{E}=-\mathbf{p}_{0} \cdot \mathbf{E}=-p_{0} E \cos \theta
$$

where $p_{0}=\left|\mathbf{p}_{0}\right|, E=|\mathbf{E}|$. For noninteracting cells and moments, classical Boltzmann statistics can be used to express the probability of dipoles occupying certain energy states. Letting $k_{B}$ denote Boltzmann's constant, the thermal energy is $k_{B} T$ and the probability that a dipole occupies the energy state $\mathcal{E}$ is

$$
\mu(\mathcal{E})=C e^{-\mathcal{E} / k_{B} T}
$$

The parameter $C$ is later specified to ensure that integration over all possible configurations yields the total number of moments per unit volume $N$. We now consider three models based on differing assumptions concerning the moment orientations.

\section{Langevin Model}

The first model is obtained under the assumption that the material is isotropic and a cell's orientation can be in any direction. This situation is equivalent to a dielectric fluid [13] where the dipole moments are free to move in any direction upon application of the electric field and yields the Langevin model commonly employed in ferromagnetic applications. 
Under the assumption of an isotropic material, the a priori number of moments between $\theta$ and $\theta+d \theta$ is proportional to the surface area $2 \pi \sin \theta d \theta$ on a unit sphere. With the probability of occupying that state given by (3), the number of moments in this configuration can be expressed as

$$
d N=2 \pi \sin \theta d \theta C e^{p_{0} E \cos \theta / k_{B} T} .
$$

To evaluate $C$, we note that the integration of $d N$ over all possible configurations must equal the total number of moments per unit volume $N$; hence

$$
\begin{aligned}
C & =\frac{N}{2 \pi \int_{0}^{\pi} e^{p_{0} E \cos \theta / k_{B} T} \sin \theta d \theta} \\
& =\frac{N}{\frac{4 \pi k_{B} T}{p_{0} E} \sinh \left(\frac{p_{0} E}{k T}\right)} .
\end{aligned}
$$

Because each cell contributes $p_{0} \cos \theta$ to the polarization, the total polarization is

$$
\begin{aligned}
P & =2 \pi \int_{0}^{\pi} p_{0} C e^{p_{0} E \cos \theta / k_{B} T} \cos \theta \sin \theta d \theta \\
& =r \pi p_{0} C\left[\frac{k_{B} T}{p_{0} E} \cosh \left(\frac{p_{0} E}{k_{B} T}\right)-\left(\frac{k_{B} T}{p_{0} E}\right)^{2} \sinh \left(\frac{p_{0} E}{k_{B} T}\right)\right] .
\end{aligned}
$$

Using (4) to eliminate $C$, we obtain the Langevin relation

$$
P=p_{0} N\left[\operatorname{coth}\left(\frac{p_{0} E}{k_{B} T}\right)-\frac{k_{B} T}{p_{0} E}\right]
$$

between the field and polarization.

\section{Ising Spin Model}

The second model examined in this section is the Ising spin model which is derived under the assumption that only two orientations are possible in one cell: one in the direction of the electric field and the other in the direction directly opposite the field. Letting $N_{+}$denote the number of cells with the same orientation as the field and $N_{-}$denote the number of cells with opposing orientation, the total number of cells is

$$
N=N_{+}+N_{-}
$$

The application of Boltzmann's equation then yields the expressions

$$
N_{+}=C e^{-p_{0} E / k_{B} T} \quad, \quad N_{-}=C e^{p_{0} E / k_{B} T}
$$

for the distribution of cells in each orientation. Combining (6) and (7), we obtain

$$
N=2 C \cosh \left(\frac{p_{0} E}{k_{B} T}\right)
$$

which relates $N$ and $C$ for the Ising spin model. The polarization is the sum of each cell's contribution

$$
P=-p_{0} N_{+}+p_{0} N_{-}=2 p_{0} C \sinh \left(\frac{p_{0} E}{k_{B} T}\right)
$$


Using (8) to eliminate the parameter $C$ yields the Ising spin model

$$
P=p_{0} N \tanh \left(\frac{p_{0} E}{k_{B} T}\right)
$$

relating $P$ and $E$. This form of the anhysteretic polarization was used by Hom and Shankar in the development of a constitutive model for electrostrictive relaxor ferroelectrics [21, 22]. The reader is referred to [25, pages 214-215] for a discussion of the quantum mechanical ramifications of the Ising spin model for ferromagnetic materials.

\section{Preferred Orientation Model}

Neither the Langevin model nor the Ising spin model completely represents the actual situation in a ferroelectric lattice due to the fixed number of orientations attained by the cell. Since a polychrystalline ceramic has a random distribution of grains, these orientations should also be random. For our third model, which we call the Preferred Orientation model, we assume that a cell has two possible orientations that have opposite directions like the Ising spin model. However, the axis of orientation $\theta$ is uniformly distributed to reflect the random distribution of the grains. For cells with a given axis of orientation, the distribution is

$$
N^{+}(\theta)=C(\theta) e^{p_{0} E \cos \theta / k_{B} T}
$$

and

$$
N^{-}(\theta)=C(\theta) e^{-p_{0} E \cos \theta / k_{B} T}
$$

where $N^{+}(\theta)$ and $N^{-}(\theta)$ are the number of cells in the two possible orientations. The total number of cells with that axis is

$$
N(\theta)=2 C(\theta) \cosh \left(p_{0} E \cos \theta / k_{B} T\right)=\frac{N}{4 \pi}
$$

where $N$ again denotes the total number of cells in the lattice. The polarization for the lattice is then

$$
P=2 \pi \int_{0}^{\pi}\left[N^{+}(\theta)-N^{-}(\theta)\right] p_{0} \cos \theta \sin \theta d \theta
$$

which simplifies to

$$
P=\frac{p_{0} N}{2} \int_{0}^{\pi} \tanh \left(p_{0} E \cos \theta / k_{B} T\right) \cos \theta \sin \theta d \theta
$$

through the use of (10) through (12). Performing the integration yields the formula

$$
\begin{aligned}
P=\frac{p_{0} N}{2}\{ & \frac{k_{B} T}{E p_{0}}\left[\log \left(1+e^{-2 E p_{0} / k_{B} T}\right)+\log \left(1+e^{2 E p_{0} / k_{B} T}\right)\right] \\
& \left.+\frac{1}{2}\left(\frac{k_{B} T}{E p_{0}}\right)^{2}\left[\operatorname{Li}\left(-e^{2 E p_{0} / k_{B} T}\right)-\operatorname{Li}\left(-e^{-2 E p_{0} / k_{B} T}\right)\right]\right\}
\end{aligned}
$$

where Li represents the dilogarithm function given by

$$
\operatorname{Li}(z)=\int_{z}^{0} \frac{\log (1-x)}{x} d x
$$

The model (13) provides a characterization of the $E-P$ relation which incorporates attributes of both the Langevin and Ising spin models. 


\section{Macroscopic Relations}

The three models (5), (9) and (13) are formulated in terms of microscopic parameters such as $p_{0}$ and $N$. To obtain macroscopic versions for use as constitutive laws, the relations must be normalized to reflect the bulk properties of the material. We first assume that all three models saturate to a common polarization $P_{s}$. Secondly, we assume that they exhibit the same initial permittivity

$$
\epsilon=\frac{P_{s} E_{0} T}{E T_{c}},
$$

where $E_{0}$ and $T_{c}$ are a scaling electric field and the Curie temperature, respectively. Under these assumptions, the dielectric functions for the Langevin, Ising spin and Preferred Orientation models are respectively given by

$$
\begin{aligned}
& P=P_{s}\left[\operatorname{coth}\left(\frac{3 T_{c} E}{E_{0} T}\right)-\frac{E_{0} T}{3 T_{c} E}\right] \\
& P=P_{s} \tanh \left(\frac{T_{c} E}{E_{0} T}\right)
\end{aligned}
$$

and

$$
\begin{aligned}
P=P_{s}\left\{\frac{2 E_{0} T}{3 E T_{c}}\left[\log \left(1+e^{-3 E T_{c} / E_{0} T}\right)+\log \left(1+e^{3 E T_{c} / E_{0} T}\right)\right]\right. \\
\left.+\frac{2}{9}\left(\frac{E_{0} T}{E T_{c}}\right)^{2}\left[\operatorname{Li}\left(-e^{3 E T_{c} / E_{0} T}\right)-\operatorname{Li}\left(-e^{-3 E T_{c} / E_{0} T}\right)\right]\right\} .
\end{aligned}
$$

The relative behavior of the three models is illustrated in Figure 2. The Langevin model saturates the slowest since its cells have the most freedom in selecting orientations that are partially aligned with the electric field. The Ising Spin model saturates more quickly since the cells have very little freedom and must completely orient in the direction of the field while the Preferred Orientation

model provides a compromise between the other two models. We note that the Langevin and Ising spin models have equivalent first-order terms but differ in the third and higher order terms.

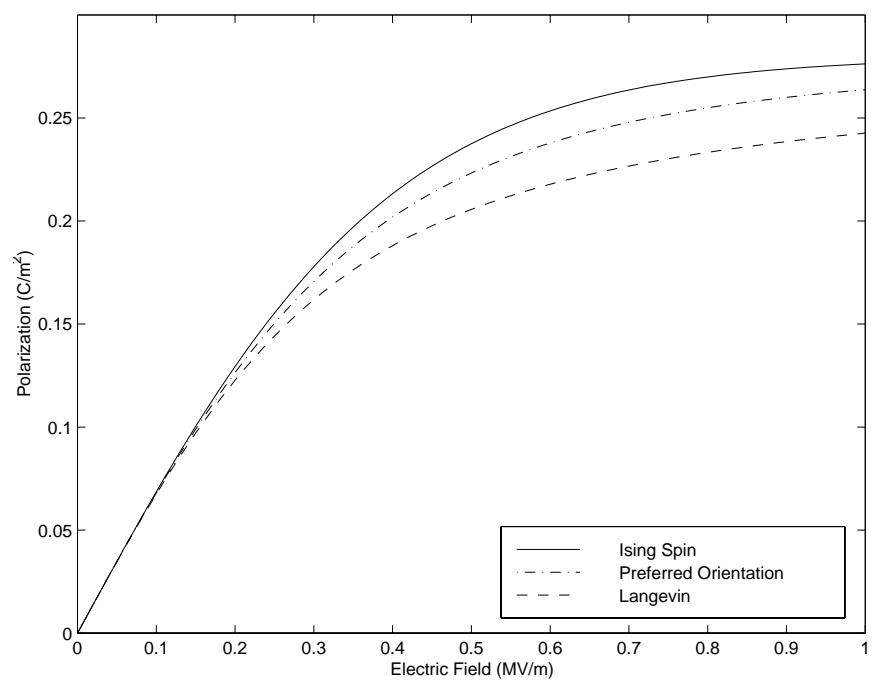

Figure 2. Ising spin, Preferred Orientation and Langevin models for the anhysteretic polarization with no cell interactions. 


\subsection{Cell Interactions}

The theoretical development of the previous subsection ignores mutual interaction between the polar moments of the cells. These interactions are often significant and can lead to both second-order phase transitions of the type described by Devonshire [10] and domain nucleation and growth. To incorporate the coupling due to neighboring domains, one typically considers the effective field acting on the cell rather than focusing solely on the applied field $E$.

\section{Effective Fields}

The issues concerning the computation of an effective field are illustrated by the Clausius-Mossotti equation which models dipole interactions in dielectric fluids and certain dielectric solids [29]. As detailed in $[1,13]$, this model quantifies the changes in the local electric field due to the polarization in the surrounding dielectric through the approximation of the field inside a sphere with a surface charge equivalent to the polarization. The resulting effective field is

$$
E_{e}=E+\frac{1}{3 \epsilon_{0}} P
$$

where $\epsilon_{0}$ denotes the permittivity of free space. Through the incorporation of the polarization component, the effective field accounts for mutual cell interactions and thus provides a better approximation of the field which actually influences individual cells.

For materials such as $\mathrm{BaTiO}_{3}$, however, the validity of the Clausius-Mossotti is doubtful [9] which has prompted the development of more general effective field models for dielectric solids [1, 39]. Hom and Shankar [22] have also extended this analysis to ferroelectric materials whose anhysteretic behavior is modeled by the Ising spin model. Under the assumption that it is energetically favorable for neighboring cells to have the same spin, the effective field in the absence of an applied stress is

$$
E_{e}=E+\alpha P
$$

where $\alpha \equiv E_{0} / P_{s}$. This model can be extended to materials subjected to an external stress $\sigma$ through consideration of the effective field

$$
E_{e}=E+\alpha P+2 Q_{33} P \sigma
$$

where $Q_{33}$ again denotes the longitudinal electrostrictive coefficient.

For the development of subsequent domain wall theory, it will be necessary to relate the effective electric field $E_{e}$ to a corresponding field $D_{e}$. For general fields, $D$ is related to $E$ and $P$ through the equation

$$
D=\epsilon_{0} E+P,
$$

where $\epsilon_{0}$ denotes the free space permittivity, or the relation

$$
D=\epsilon E
$$

where $\epsilon$ is the permittivity of the substance. It should be noted that for ferroelectric materials, the permittivity $\epsilon$ is in general both nonlinear and multivalued due to hysteresis. For such materials, the relation (19) has limited value as a constitutive relation until $\epsilon$ is characterized. When quantifying hysteresis losses in the material, we employ the field

$$
D_{e}=\epsilon_{0}\left(E+\alpha P+2 Q_{33} P \sigma\right) .
$$


This definition is analogous to the expression $B_{e}=\mu_{0}(H+\alpha M)=\mu_{0} H_{e}$ employed for ferromagnetic materials (e.g., see [26, page 1266] along with [4, 27, 28, 47]) where $H_{e}$ and $B_{e}$ respectively denote the effective magnetic field and induction.

\section{Macroscopic Constitutive Relations and Phase Transitions}

Because the effective field $E_{e}$ incorporates the contributions due to interdomain coupling and applied stresses, we will employ it when describing the general model for the anhysteretic polarization and developing constitutive relations for the material. The consideration of the effective field $E_{e}$ in (14) yields the Langevin model

$$
P=P_{s}\left[\operatorname{coth}\left(\frac{3 T_{c} E_{e}}{E_{0} T}\right)-\frac{E_{0} T}{3 T_{c} E_{e}}\right]
$$

and the Ising spin model

$$
P=P_{s} \tanh \left(\frac{T_{c} E_{e}}{E_{0} T}\right)
$$

for the anhysteretic polarization, with analogous treatment in the Preferred Orientation Model. In the case of constant temperature, the anhysteretic polarization provided by the Langevin and Ising spin models can be expressed as

$$
P=P_{s}\left[\operatorname{coth}\left(\frac{E_{e}}{a}\right)-\frac{a}{E_{e}}\right]
$$

and

$$
P=P_{s} \tanh \left(\frac{E_{e}}{a}\right)
$$

where $a=\frac{E_{0} T}{3 T_{c}}$ or $a=\frac{E_{0} T}{T_{c}}$ are constants which must be estimated for a given material or device.

To obtain a constitutive dielectric equation commensurate with the mechanical constitutive relation (1), the characterization must be polarization-based. This means that the dielectric relations developed in this section must be inverted to obtain the electric field as a function of the polarization. Of the three models, this can be accomplished in closed form only for the Ising spin model. For that model, inversion of the relation (22) and combination with the mechanical relation yields the constitutive equations

$$
\begin{aligned}
& \sigma=Y\left(e-Q_{33} P^{2}\right) \\
& E=-2 Q_{33} P \sigma-\frac{E_{0}}{P_{s}} P+\frac{E_{0} T}{T_{c}} \operatorname{arctanh}\left(\frac{P}{P_{s}}\right)
\end{aligned}
$$

so that $\mathcal{F}$ in $(1)$ is given by

$$
\mathcal{F}(T, P)=-\frac{E_{0}}{P_{s}} P+\frac{E_{0} T}{T_{c}} \operatorname{arctanh}\left(\frac{P}{P_{s}}\right) .
$$

Finally, the consideration of the dielectric constitutive equation in (25) illustrates the nature of phase transitions in the material. Consider the relation

$$
\frac{P}{P_{s}}=\frac{T}{T_{c}} \operatorname{arctanh}\left(\frac{P}{P_{s}}\right)
$$

which results in the absence of an applied field $(E=0)$ and stress $(\sigma=0)$. The left hand side represents the force driving cells to have the same orientation while the right hand side represents 
the tendency for random orientation due to thermal agitation. When the temperature is above the transition temperature $T_{c}$, the only solution to (26) is $P=0$ as shown in Figure 3a. In this case, thermal agitation overpowers the ordering force and the random orientation of the dipoles leads to zero average polarization in the ceramic. Below the transition temperature, three polarization solutions exist for (26). The solution $P=0$ is unstable while the other two solutions are stable and represent a spontaneous nonzero polarization. These nonzero solutions determine the physical behavior of the ferroelectric materials at temperatures below the Curie point. For a fixed temperature $T$ below the Curie point $T_{c}$, the relation between an input field and the resulting polarization is plotted in Figure 3b. The instability at $P=0$ is noted in both the initial polarization and the behavior at the coercive field values.

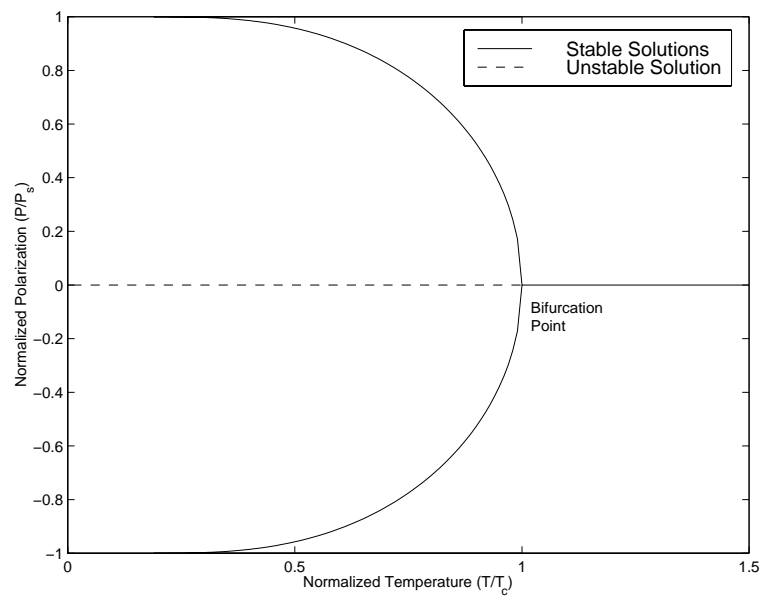

(a)

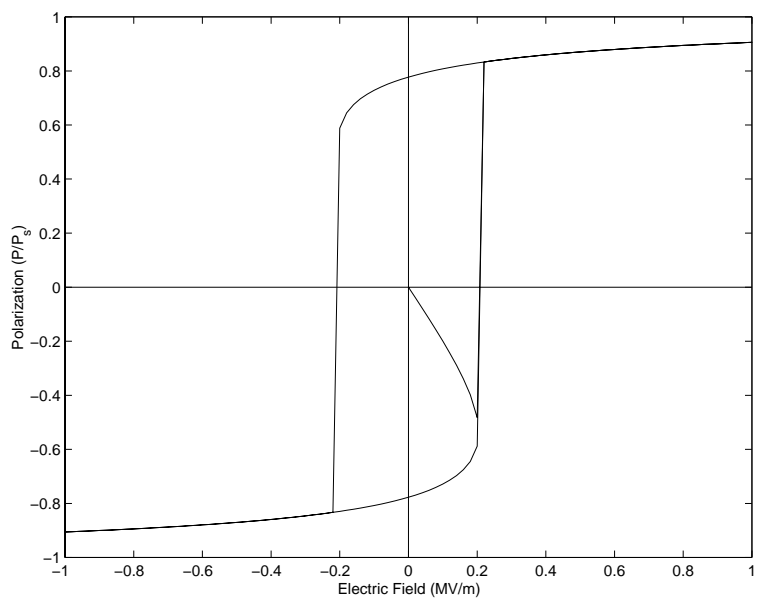

(b)

Figure 3. (a) The phase transition predicted by the Ising spin model with cooperative interaction between cells; (b) Relation between an input field $E$ and the polarization of PMN-PT for $T<T_{c}$.

\section{Domain Wall Theory}

The relations (23) or (24), in combination with the effective field relations (16) or (17), quantity the anhysteretic relation between the electric field $E$ input to the material and the resulting polarization $P$. For most values of the parameters $\alpha$ and $a$, the relation is single-valued. As illustrated in Figure $3 \mathrm{~b}$, however, one can obtain a multivalued relation for certain parameter choices. While this latter configuration produces a curve which quantifies certain aspects of hysteresis, the transition from one saturation region to the other is much steeper than that observed in typical ferroelectric applications.

The anhysteretic domain process is dependent upon unimpeded domain wall movement and is reversible and conservative. As illustrated by the experimental data in [22], this is often not the case and the material exhibits hysteresis and nonreversible behavior. In this model, we incorporate this hysteresis through consideration of energy losses at domain wall pinning sites.

\subsection{Pinning Energy}

As indicated in the first section, all ferroelectric materials exhibit material nonhomogeneities, voids, and stress variations which are energetically favorable for domain wall formation and pinning. Because these inclusions inhibit domain wall movement, they lead to energy loss as the material is 
cycled through a full E-P loop. To incorporate the hysteresis observed under constant temperature, quasistatic operating conditions, we quantify the reversible and irreversible domain wall dynamics exhibited at pinning sites. This theory follows closely that derived by Jiles and Atherton for ferromagnetic materials [27].

We consider first the energy required to break the pinning site and hence move the domain wall. As detailed in [7], a pinning site is broken when sufficient energy is provided to overcome a local energy barrier. Consider the domain configuration depicted in Figure 4 in which two domains are separated by a domain wall situated at a pinning site. The dipole moments per unit volume in the two domains are denoted by $\mathbf{p}$ and $\mathbf{p}^{\prime}$ with $\mathbf{p}$ assumed to be aligned with the effective electric field $\mathbf{E}_{e}$ (this assumption simplifies the discussion and produces the same final model as that determined with a general configuration). The angle between the moments is denoted by $\theta$.

For ferromagnetic materials, Jiles and Atherton hypothesized that the energy required to overcome the pinning sites, and hence move the domain wall, is proportional to the change in energy required to align the magnetic moments with the field [27]. We make the same assumption for ferroelectric materials.

For a dipole moment $\mathbf{p}$, the energy due to the effective field is

$$
\mathcal{E}=-\mathbf{p} \cdot \mathbf{E}_{e}
$$

so that the change in energy required to rotate the moments $\mathbf{p}^{\prime}$ in the direction of the field is

$$
\Delta \mathcal{E}=-\mathbf{p} \cdot \mathbf{E}_{e}+\mathbf{p}^{\prime} \cdot \mathbf{E}_{e} .
$$

Under the assumption that the energy $\mathcal{E}_{\text {pin }}$ required to break pinning sites is proportional to this change, it follows that

$$
\mathcal{E}_{\text {pin }}=-p E_{e} c_{1}(1-\cos \theta)
$$

where $p=|\mathbf{p}|, E_{e}=\left|\mathbf{E}_{e}\right|$ and $c_{1}$ is the constant of proportionality. To eliminate $c_{1}$, we let $\mathcal{E}_{\pi}$ denote the energy for $180^{\circ}$ domain walls and note that $\mathcal{E}_{\pi}=2 p E_{e} c_{1}$. This implies that

$$
\mathcal{E}_{\text {pin }}=-\frac{1}{2} \mathcal{E}_{\pi}(1-\cos \theta) \text {. }
$$

The relation (28) quantifies the energy required to break a single pinning site. To obtain a corresponding macroscopic relation which quantifies the behavior of the bulk material, we employ the techniques of Jiles and Atherton [27] and let $n$ denote the average density of pinning sites and $\left\langle\mathcal{E}_{\pi}\right\rangle$ denote the average energy for $180^{\circ}$ walls. The energy required to break pinning sites if a domain wall of area $A$ moves a distance $x$ is then

$$
\mathcal{E}_{\text {pin }}(x)=-\int_{0}^{x} \frac{n\left\langle\mathcal{E}_{\pi}\right\rangle}{2}(1-\cos \theta) A d x .
$$

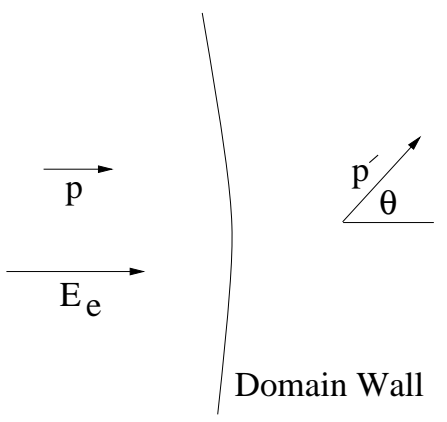

Figure 4. Domain wall and orientation of dipole moments $\mathbf{p}, \mathbf{p}^{\prime}$ and electric field $\mathbf{E}_{e}$. 
Because the polarization is the total contribution due to dipole moments in a given volume, the change in polarization due to the reorientation of moments is

$$
d P=-p(1-\cos \theta) A d x .
$$

With the definition $k=\frac{n\left\langle\mathcal{E}_{\pi}\right\rangle}{2 p},(28)$ and $(29)$ can be combined to yield the expression

$$
\mathcal{E}_{\text {pin }}(P)=-k \int_{0}^{P} d P
$$

for the energy required to break pinning sites. Note that this relation provides a macroscopic description due to the averaging of domain density and energy in the definition of $k$.

Completely analogous arguments follow for $90^{\circ}$ domain walls as well as materials which exhibit rhombohedral symmetries $\left(71^{\circ} / 109^{\circ}\right.$ domain walls) with the final expressions for the pinning energy differing only in the values of $k$. Because the number of pinning sites and average energy are unknown, the parameter $k$ must be estimated for any of these domain configurations through a least squares fit to data.

\subsection{Work in the Polarization Process}

To quantify the irreversible and reversible polarization processes, we must determine the work required to attain a specified polarization level. This can be formally obtained from classical expressions for the work required to configure a specified charge distribution or derived from first principles of electrostatic field theory.

As detailed in [46], if $\rho$ denotes a charge density, then the work necessary to produce a change $\delta \rho$ is given by

$$
\delta W=\int_{V} \phi \delta \rho d V
$$

where $\phi$ is the associated scalar potential. The application of Maxwell's equations then yields

$$
\delta W=\int_{V} \mathbf{E} \cdot \delta \mathbf{D} d V
$$

so that the change in energy density is $\mathbf{E} \cdot \delta \mathbf{D}$. This yields the classical expression

$$
w=\int_{0}^{\mathbf{D}} \mathbf{E} \cdot d \mathbf{D}
$$

for the energy required to create the field at a point.

For this analysis, we are interested in the work required to attain a specified polarization level through the application of the interaction field $\mathbf{D}_{e}$. Such a relation can be formally obtained by employing the relation (18) in (31) to obtain

$$
w=\frac{1}{\epsilon_{0}} \int_{0}^{\mathbf{D}} \mathbf{D} \cdot d \mathbf{D}-\frac{1}{\epsilon_{0}} \int_{0}^{\mathbf{D}} \mathbf{P} \cdot d \mathbf{D} .
$$

Over a full cycle, the first term on the right hand side is zero so that the losses are given by

$$
w=-\frac{1}{\epsilon_{0}} \oint \mathbf{P} \cdot d \mathbf{D} .
$$

Employing the interaction field $\mathbf{D}_{e}$, one is then left with the energy functional

$$
w=\frac{1}{\epsilon_{0}} \int_{0}^{\mathbf{D}_{e}} \mathbf{P} \cdot d \mathbf{D}_{e}
$$

for specifying hysteresis losses over a portion of the polarization cycle. Further details regarding the derivation of the functional (32) are provided in Appendix A. We point out that the energy functional (32) is analogous to that employed by Jiles and Atherton [27] in their magnetization model. 


\subsection{Irreversible Polarization}

As detailed by Chen and Wang [7], the dielectric properties of ferroelectric materials are influenced by both the bending behavior of domain walls pinned by inclusions and the long-range movement of domain walls when local energy barriers are overcome. We will denote the respective polarization components as the reversible polarization and irreversible polarization and employ the strategy of Jiles and Atherton to model the two phenomena.

To characterize the irreversible polarization, we note that the polarization energy for a given effective field level can be expressed as that observed in the ideal (anhysteretic) case minus losses necessary to overcome local energy barriers and hence break pinning sites. Employing the relation (32) for the energy and (30) for the losses at pinning sites, this yields

$$
\frac{1}{\epsilon_{0}} \int_{0}^{D_{e}} P_{i r r}\left(E_{e}\right) d D_{e}=\frac{1}{\epsilon_{0}} \int_{0}^{D_{e}} P_{a n}\left(E_{e}\right) d D_{e}-\int_{0}^{D_{e}} k \frac{d P_{i r r}}{d D_{e}} d D_{e}
$$

where $P_{i r r}$ denotes the irreversible polarization and $P_{a n}$ is the anhysteretic polarization given by (23) or (24) for the effective field $E_{e}=E+\alpha P_{i r r}$. Differentiation then yields

$$
P_{i r r}=P_{a n}-\delta \epsilon_{0} k \frac{d P_{i r r}}{d D_{e}}
$$

where the parameter $\delta=\operatorname{sign}(d E)$ ensures that the energy required to break pinning sites always opposes changes in the polarization. To obtain an expression which facilitates numerical implementation, we reformulate (33) to obtain

$$
\begin{aligned}
P_{a n}-P_{i r r} & =\delta \epsilon_{0} k \frac{d P_{i r r}}{d D_{e}} \\
& =\delta k \frac{d P_{i r r}}{d E}\left(\frac{1}{1+\alpha \frac{d P_{i r r}}{d E}}\right)
\end{aligned}
$$

from which it follows that

$$
\frac{d P_{i r r}}{d E}=\frac{P_{a n}-P_{i r r}}{\delta k-\alpha\left(P_{a n}-P_{i r r}\right)} .
$$

We note that for certain materials and operating regimes, the indiscriminate use of the differential equation (34) can lead to nonphysical solutions when the field is reversed in the saturation region of the hysteresis loop (near the tip in the first and third quadrants). Specifically, for very wide hysteresis loops of the type illustrated in Figure 1, direct solution of (34) yields a negative differential electric susceptibility $\frac{d P}{d E}$ when $E$ is reversed and the magnitude of $P$ is less than that of the global anhysteretic (see Figure 6). This phenomenon is not observed experimentally and the model is modified slightly for these situations. As noted in [28] where the analogous ferromagnetic phenomenon is analyzed, domain walls remain pinned when the field is initially reversed and the primary change in polarization is due to the reversible relaxation of bulged domain walls. Hence we enforce the condition $\frac{d P_{i r r}}{d E}=0$ until the polarization crosses the anhysteretic curve. This produces the more physically realistic expression

$$
\frac{d P_{i r r}}{d E}=\widetilde{\delta} \frac{P_{a n}-P_{i r r}}{k \delta-\alpha\left(P_{a n}-P_{i r r}\right)}
$$

where

$$
\widetilde{\delta}=\left\{\begin{array}{l}
1,\left\{d E>0 \text { and } P>P_{a n}\right\} \text { or }\left\{d E<0 \text { and } P<P_{a n}\right\} \\
0, \text { otherwise } .
\end{array}\right.
$$

We point out that this additional constrain is unnecessary for most sigmoid hysteresis curves and is required only for materials which exhibit large hysteresis losses and are driven to full saturation. 


\subsection{Reversible Polarization}

A basic tenant of domain and domain wall theory for ferroelectric materials is the observation that walls exhibit bulging and motion before manifesting irreversible effects [7, 12, 30]. While this reversible component of the polarization is typically smaller than the irreversible component, the effects are still significant and must be incorporated to obtain an accurate model. The development of the reversible polarization follows closely the theory provided by Jiles and Atherton [27] for ferromagnetic materials.

To quantify the reversible polarization $P_{r e v}$, we first consider the energy required to change the polarization from $P_{a n}\left(E_{e}\right)$ to $P\left(E_{e}\right)$, where again $E_{e}=E+\alpha P$, at a fixed field level $E$. From (27), it follows that the change in energy per unit volume required to attain this change is

$$
\Delta \mathcal{E}_{V}=-\left[\mathbf{P} \cdot \mathbf{E}_{e}-\mathbf{P}_{a n} \cdot \mathbf{E}_{e}\right] .
$$

Under the assumption that $|\mathbf{P}|=\left|\mathbf{P}_{a n}\right|=P$ and employing the law of cosines, this yields

$$
\begin{aligned}
\Delta \mathcal{E}_{V} & =P E_{e}(1-\cos \theta) \\
& =\frac{E_{e}}{2 P}\left(P-P_{a n}\right)^{2}
\end{aligned}
$$

where $\left(P-P_{a n}\right)=\left|\mathbf{P}-\mathbf{P}_{a n}\right|$. For fixed field values, the force on the domain walls is then

$$
F=\frac{\alpha\left[\epsilon_{0}-\epsilon(E)\right]-1}{\epsilon(E)-\epsilon_{0}}\left(P-P_{a n}\right)
$$

since $P=\left[\epsilon(E)-\epsilon_{0}\right] E$. Neglecting higher order terms and denoting the multiplicative constant by $k_{1}$ then yields the expression

$$
\mathcal{P}=k_{1}\left(P-P_{a n}\right),
$$

for the pressure on the domain wall.

To obtain the reversible polarization due to wall movement in response to this pressure, we need to approximate the displaced volume. To specify the geometry, we consider a domain wall between two pinning sites separated by a distance of $2 y$. The wall is assumed to bow an amount $x$ in response to the applied pressure $\mathcal{P}$ with a resulting radius of curvature $r$ (see Figure 5 ).

If we let $\mathcal{E}_{S}$ denote the surface energy of the domain wall, then the pressure can be expressed as

$$
\mathcal{P}=\frac{2 \mathcal{E}_{S}}{r}
$$

Using techniques analogous to those employed in Jiles and Atherton [27], it then follows that

$$
\begin{aligned}
x & =r-\sqrt{r^{2}-y^{2}} \\
& =\frac{2 \mathcal{E}_{S}}{\mathcal{P}}-\sqrt{\left(\frac{2 \mathcal{E}_{S}}{\mathcal{P}}\right)^{2}-y^{2}} \\
& \approx \frac{y^{2} \mathcal{P}}{4 \mathcal{E}_{S}} \quad \text { (Binomial Theorem) } \\
& =\frac{y^{2} k_{1}}{4 \mathcal{E}_{S}}\left(P_{a n}-P\right) .
\end{aligned}
$$


Under the assumption that the wall displaces a spherical solid angle, the change in volume is given by $\Delta V=\frac{\pi}{6} x\left(3 y^{2}+x^{2}\right)$. For the case in which separated domains are parallel and antiparallel to the applied field, the reversible polarization is

$$
\begin{aligned}
P_{\text {rev }} & =2 \Delta V p \\
& =\frac{p \pi y^{4} k_{1}}{4 \mathcal{E}_{S}}\left(P_{a n}-P\right)+\frac{p \pi}{3}\left(\frac{y^{2} k_{1}}{4 \mathcal{E}_{S}}\right)^{3}\left(P_{a n}-P\right)^{3} .
\end{aligned}
$$

Consideration of first-order terms then yields the expression

$$
P_{\text {rev }}=c_{1}\left(P_{\text {an }}-P\right)
$$

for the reversible polarization. The constant $c_{1}=\frac{p \pi y^{4} k_{1}}{4 \mathcal{E}_{S}}$ must be estimated through a least squares fit to data since the components $p, y, k_{1}$ and $\mathcal{E}_{S}$ are unknown.

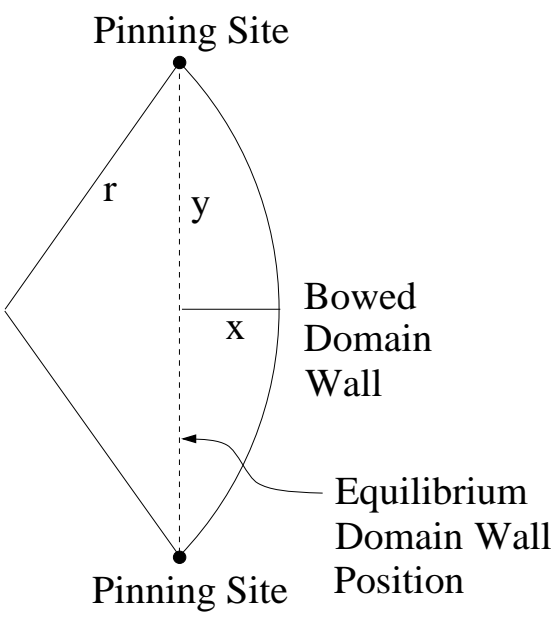

Figure 5. Geometry of the domain wall with reversible bowing (from Jiles and Atherton [27]).

\subsection{Total Polarization}

The total polarization can now be specified through the following algorithm. For a given field $E$, polarization $P$ and constant stress $\sigma$, the effective field and anhysteretic polarization are given by (17) and (23) or (24), respectively. The differential equation (35) is then integrated to compute the irreversible polarization $P_{i r r}$. Finally, the total polarization $P$ is given by the sum

$$
P=P_{\text {irr }}+P_{\text {rev }} .
$$

The reversible polarization $P_{\text {rev }}$ can be specified either by (37) or by the expression

$$
P_{\text {rev }}=c\left(P_{a n}-P_{\text {irr }}\right)
$$

where $c=\frac{c_{1}}{1+c_{1}}$. For the remainder of this development, we will employ the expression (39) since it permits the separation of the reversible and irreversible polarizations.

The full time-dependent model leading from an input field $E(t)$ to the output polarization $P(t)$ is summarized in Algorithm 1 with properties of the model parameters summarized in Table 1. The 
use of the Langevin model yields the anhysteretic polarization (iia) whereas the Ising spin model yields (iib).

The model in the form summarized in Algorithm 1 is typically employed when characterizing a material or device. For control applications, however, it can be advantageous to express the output polarization directly in terms of the input field. When the Ising spin model is used to characterize the anhysteretic magnetization, the expressions (24), (35), (38) and (39) can be consolidated to yield

$$
\begin{aligned}
\frac{d P}{d E} & =(1-c) \frac{d P_{i r r}}{d E}+c \frac{d P_{a n}}{d E} \\
& =\widetilde{\delta}(1-c) \frac{P_{a n}-P_{i r r}}{k \delta-\alpha\left(P_{a n}-P_{i r r}\right)}+c \frac{d P_{a n}}{d E} \\
& =\frac{\widetilde{\delta}\left(P_{a n}-P\right)}{k \delta-\frac{\alpha}{1-c}\left(P_{a n}-P\right)}+c \frac{d P_{a n}}{d E} \\
& =\frac{\widetilde{\delta}\left[P_{s} \tanh \left(\frac{E+\alpha P}{a}\right)-P\right]}{k \delta-\tilde{\alpha}\left[P_{s} \tanh \left(\frac{E+\alpha P}{a}\right)-P\right]}+\frac{c P_{s}}{a} \operatorname{sech}^{2}\left(\frac{E+\alpha P}{a}\right)\left(1+\alpha \frac{d P}{d E}\right)
\end{aligned}
$$

where $\tilde{\alpha}=\frac{\alpha}{1-c}$. The magnetization at a given field level is then specified by the solution to the differential equation

$$
\begin{aligned}
& \frac{d P}{d E}=\mathcal{G}(E, P) \\
& P\left(E_{0}\right)=P_{0}
\end{aligned}
$$

where

$$
\begin{aligned}
& \mathcal{G}(E, P)=\frac{1}{1-\frac{c P_{s} \alpha}{a} \operatorname{sech}^{2}\left(\frac{E+\alpha P}{a}\right)} \cdot\left\{\frac{\widetilde{\delta}\left[P_{s} \tanh \left(\frac{E+\alpha P}{a}\right)-P\right]}{k \delta-\tilde{\alpha}\left[P_{s} \tanh \left(\frac{E+\alpha P}{a}\right)-P\right]}\right. \\
& \left.+\frac{c P_{s}}{a} \operatorname{sech}^{2}\left(\frac{E+\alpha P}{a}\right)\right\} .
\end{aligned}
$$

If one employs the Langevin expression (23) rather than the Ising spin relation (24) for the anhysteretic, the function $\mathcal{G}$ is given by

$$
\begin{array}{r}
\mathcal{G}(E, P)=\frac{1}{1+\frac{c P_{s} \alpha}{a}\left[\operatorname{csch}^{2}\left(\frac{E+\alpha P}{a}\right)-\left(\frac{a}{E+\alpha P}\right)^{2}\right]} \cdot\left\{\frac{\widetilde{\delta}\left[P_{s} \mathcal{L}\left(\frac{E+\alpha P}{a}\right)-P\right]}{k \delta-\tilde{\alpha}\left[P_{s} \mathcal{L}\left(\frac{E+\alpha P}{a}\right)-P\right]}\right. \\
\left.-\frac{c P_{s}}{a}\left[\operatorname{csch}^{2}\left(\frac{E+\alpha P}{a}\right)-\left(\frac{a}{E+\alpha P}\right)^{2}\right]\right\}
\end{array}
$$

where the Langevin function is defined by

$$
\mathcal{L}(z) \equiv \operatorname{coth}(z)-\frac{1}{z} .
$$

As detailed in [49], where the analogous magnetization model is considered, the formulation (40) is directly amenable to inversion. This provides a mechanism for obtaining an inverse compensator for linear control design. 


\section{Remark 1:}

The previous derivation has involved two aspects of the anhysteretic polarization, namely, the global anhysteretic polarization and local anhysteretic polarization curves. To facilitate implementation, we summarize pertinent attributes of the two components.

\section{Global Anhysteretic:}

The global anhysteretic represents the polarization attained in the absence of pinning sites with zero remanent polarization. As detailed in Section 2, the global anhysteretic polarization depends upon the applied field $E$ as indicated in the Ising spin model $P_{a n}=P_{s} \tanh \left(\left(E+\alpha P_{a n}\right) / a\right)$ or Langevin model $P_{a n}=P_{s} \mathcal{L}\left(\left(E+\alpha P_{a n}\right) / a\right)$. The slope of the curve $P_{a n}$ is determined by the ratio of the parameters $\alpha=E_{0} / P_{s}$ and $a=E_{0} T / T_{c}$ with sufficiently small values of $a$ (e.g., due to $T<T_{c}$ for PMN) producing the multi-valued curve plotted in Figure 3b. To illustrate the dependence of the global anhysteretic polarization on these parameters, the curves obtained with $\alpha=1.53 \times 10^{6} \mathrm{Vm} / \mathrm{C}$, $a=1.8 \times 10^{5}\left(T>T_{c}\right.$ for PMN $)$ and $\alpha=1.53 \times 10^{6} \mathrm{Vm} / \mathrm{C}, a=0.7 \times 10^{5}\left(T<T_{c}\right.$ for PMN $)$ are plotted in Figure 6a. It should be noted that the multi-valued curve obtained with the latter parameter values is the global anhysteretic obtained in Section 4 when constructing the polarization model for a PMN actuator employed below the Curie temperature.

\section{Local Anhysteretic:}

For the formulation of the irreversible polarization developed in Section 3.3, the local anhysteretic polarization is computed using the effective field $E_{e}=E+\alpha P_{i r r}$ rather than the equilibrium value $E_{e}=E+\alpha P_{a n}$ employed for the global anhysteretic. We interpret this as the hysteresis-free polarization which would result if one started at a point $\left(E, P_{i r r}\right)$ on the irreversible polarization curves and modified the field. Because the local anhysteretic incorporates the prevailing irreversible polarization, we employ it in Algorithm 1 when computing the anhysteretic response to an applied electric field.

The local anhysteretic polarization with the parameter values $\alpha=1.53 \times 10^{6} \mathrm{Vm} / \mathrm{C}, a=1.8 \times 10^{5}$ is plotted along with the global anhysteretic and total polarization in Figure $6 \mathrm{~b}$. It is noted that the local anhysteretic more closely resembles the total polarization than does the low temperature, multi-valued global anhysteretic curve depicted in Figure 6a. Finally, it should be noted that in the absence of coupling effects $(\alpha=0)$, the local and global anhysteretic curves coincide.

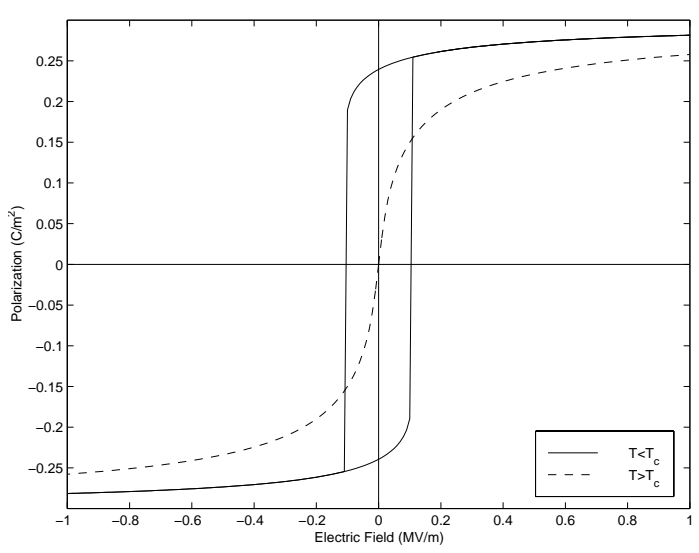

(a)

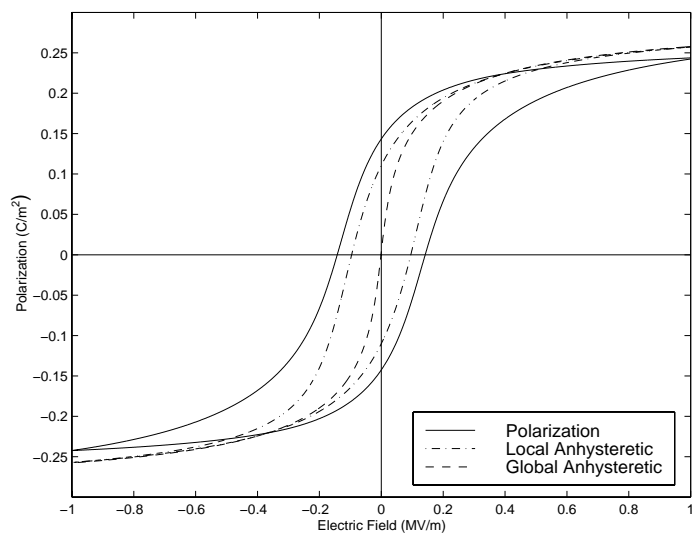

(b)

Figure 6. (a) The global anhysteretic $P_{a n}=P_{s} \mathcal{L}\left(\left(E+\alpha P_{a n}\right) / a\right), a=E_{0} T / T_{c}$, of PMN for temperatures $T<T_{c}$ and $T>T_{c}$. (b) The local anhysteretic $P_{a n}=P_{s} \mathcal{L}\left(\left(E+\alpha P_{\text {irr }}\right) / a\right)$, global anhysteretic, and total polarization of PMN for $T>T_{c}$. 
We note that in certain applications of the Jiles-Atherton model, the effective field used to define the irreversible magnetization is formulated in terms of the total magnetization rather than the irreversible magnetization. This provides an adequate approximation when reversible effects are small. A similar approximation can be considered for ferroelectric materials, in which case, the expressions (i) and (iii) in Algorithm 1 are replaced by

$$
\begin{aligned}
& \text { (i') } \quad E_{e}(t)=E(t)+\alpha P(t) \\
& \text { (iii') } \quad \frac{d P_{i r r}}{d t}(t)=\frac{d E}{d t} \cdot \frac{\tilde{\delta}\left[P_{a n}(t)-P_{i r r}(t)\right]}{k \delta-\alpha\left[P_{a n}(t)-P_{i r r}(t)\right] \frac{d P_{i r r}}{d P}} .
\end{aligned}
$$

The derivative $\frac{d P_{i r r}}{d P}$ results from expanding (33) with the total polarization employed in the effective field. For materials in which the reversible polarization is small, $\frac{d P_{i r r}}{d P} \approx 1$, and the expression (iii') is adequately approximated by (iii). This is the case for the magnetism in certain applications where the Jiles-Atherton model is employed. For ferroelectric materials in which the reversible polarization is significant, this derivative must be retained if the expressions (i') and (iii') are substituted for (i)

\begin{tabular}{|c|c|c|}
\hline (i) & $E_{e}(t)=E(t)+\alpha P_{i r r}(t)$ & \\
\hline (iia) & $P_{a n}(t)=P_{s}\left[\operatorname{coth}\left(\frac{E_{e}(t)}{a}\right)-\left(\frac{a}{E_{e}(t)}\right)\right]$ & (Langevin Model) \\
\hline (iib) & $P_{a n}(t)=P_{s} \tanh \left(\frac{E_{e}(t)}{a}\right)$ & (Ising Spin Model) \\
\hline (iii) & $\frac{d P_{i r r}}{d t}(t)=\frac{d E}{d t} \cdot \frac{\widetilde{\delta}\left[P_{a n}(t)-P_{i r r}(t)\right]}{k \delta-\alpha\left[P_{a n}(t)-P_{i r r}(t)\right]}$ & \\
\hline (iv) & $P_{r e v}(t)=c\left[P_{a n}(t)-P_{i r r}(t)\right]$ & \\
\hline$(\mathrm{v})$ & $P(t)=P_{r e v}(t)+P_{i r r}(t)$ & \\
\hline
\end{tabular}
and (iii).

Algorithm 1. Time-dependent model quantifying the output polarization $P(t)$ for $\sigma=0$. 


\begin{tabular}{c|l|l}
\hline Parameter & Physical Property & Effects on Model \\
\hline$\alpha$ & $\begin{array}{l}\text { Quantifies domain } \\
\text { interactions }\end{array}$ & $\begin{array}{l}\text { Increased values lead to steeper slopes for } \\
\text { anhysteretic and polarization curves. }\end{array}$ \\
\hline$a$ & $\begin{array}{l}\text { Shape parameter } \\
\text { for } P_{a n}\end{array}$ & Increased value decreases slope of $P_{a n} \cdot$ \\
\hline$k$ & $\begin{array}{l}\text { Average energy } \\
\text { required to break } \\
\text { pinning sites }\end{array}$ & $\begin{array}{l}\text { Increased value produces wider hysteresis } \\
\text { curve and narrower minor loop. }\end{array}$ \\
\hline $\begin{array}{l}\text { Reversibility coeffi- } \\
\text { cient }\end{array}$ & $\begin{array}{l}\text { Decrease in value leads to wider hysteresis } \\
\text { curve. }\end{array}$ \\
\hline$P_{s}$ & $\begin{array}{l}\text { Saturation } \\
\text { polarization }\end{array}$ & $\begin{array}{l}\text { Increase leads to large saturation value for } \\
\text { polarization. }\end{array}$ \\
\hline
\end{tabular}

Table 1. Physical properties and effects of the model parameters $\alpha, a, k, c, P_{s}$. The parameters $\delta=\operatorname{sign}(d E)$ and $\widetilde{\delta}$ given by (36) are computed directly from the measured field and polarization.

\section{Model Validation}

The model derived in Section 3 and summarized in Algorithm 1 provides a relation between electric fields input to a ferroelectric actuator and the resulting polarization. This model incorporates the nonlinear constitutive behavior, through either the Langevin or Ising spin relations, as well as hysteresis due to impeded domain wall movement. When coupled with a mechanical constitutive relation, such as (1), this provides a characterization of the strains produced by the material.

To illustrate the capabilities of the model, we consider the characterization of a PMN-PT-BT actuator operating in its ferroelectric range. The material was composed of $12 \% \mathrm{PT}$ and $2 \%$ BT in solid solution and had a Curie temperature of $T_{c}=45^{\circ} \mathrm{C}$. The data was collected from a stress-free sample $(\sigma=0$ in $(25))$ at $T=-20^{\circ} \mathrm{C}$. To maintain quasistatic conditions, the frequency for the input electric field was taken to be $\omega=1 \mathrm{~Hz}$ for a complete steady-state cycle.

To employ the model, the anhysteretic relations must be specified and appropriate parameters $P_{s}, a, \alpha, k$ and $c$ must be ascertained. As noted in Section 2, a variety of models for the anhysteretic polarization have been developed and employed in ferroelectric applications. For applications in which anhysteretic polarization data can be obtained (e.g., through the choice of operating temperature or the application of a large oscillating field to "break through" pinning sites), the choice of anhysteretic model can be validated through a fit to the data. For other cases, the choice of anhysteretic model can be motivated by modeling and theoretical (e.g., thermodynamic) considerations. To model the PMN-PT-BT actuator, we consider both the Langevin and Ising spin models. These models differ in their third and higher order terms which produces the difference in saturation behavior illustrated in Figure 2.

The parameters $P_{s}, a, \alpha, k$ and $c$ must then be determined for the given material. For many materials, the saturation polarization $P_{s}$ is well known and values can be specified directly. The parameters $a, \alpha, k, c$ are less easily specified, however, since they are defined in terms of material properties which are either unknown or not easily measured. Furthermore, the values of $a, \alpha, k, c$ depend to some extent on the choice of anhysteretic model since they depend upon the shape of the modeled anhysteretic and energy differences between the anhysteretic and measured data. In applications, $a, \alpha, k, c$ and in many cases $P_{s}$, are estimated through a least squares fit to data. 
The modeled polarization obtained with the Langevin relation and the parameter values $a=$ $0.70 \times 10^{5} \mathrm{C} / \mathrm{m}^{2}, \alpha=1.53 \times 10^{6} \mathrm{Vm} / \mathrm{C}, k=4.5 \times 10^{5} \mathrm{C} / \mathrm{m}^{2}, c=.80, P_{s}=.296 \mathrm{C} / \mathrm{m}^{2}$ is compared with the experimental data in Figure 7 . We reiterate that the data was collected under quasistatic conditions with one complete steady-state cycle plotted in the figure. The measured electric field data was used as input to the model for three complete cycles. While the initial polarization curve for the model is depicted here, one would typically bring the model to a polarized state before employing it for physical transducer characterization or control design. In this regime, the model very accurately quantifies the nonlinear and hysteretic behavior of the material.

The corresponding model employing the Ising spin relation is compared with the data in Figure 8. In this case, the model was computed with the parameters $a=4.2 \times 10^{5} \mathrm{C} / \mathrm{m}^{2}, \alpha=2 \times 10^{6} \mathrm{Vm} / \mathrm{C}$, $k=3.7 \times 10^{5} \mathrm{C} / \mathrm{m}^{2}, c=.65, P_{s}=.28 \mathrm{C} / \mathrm{m}^{2}$. As expected, less than $5.5 \%$ change is observed in the saturation polarization $P_{s}$. The other four parameters differ from those obtained with the Langevin relation to accommodate the different qualitative behavior of the curves. A comparison of Figures 7 and 8 indicates that the use of the Langevin relation for the anhysteretic polarization provides a somewhat better fit than the Ising spin-based model. This indicates that for this material, the Ising spin model may saturate too quickly due to the imposed requirement that cells are restricted to only two possible orientations.

The nature of the Ising spin model is further illustrated by the global anhysteretic polarization plotted in Figure 6a. As discussed in Remark 1 and noted in the figure, this curve is multivalued at temperatures $T<T_{c}$. While this curve incorporates a form of hysteresis, it neglects the energy required to break pinning sites and hence provides an overly steep transition between saturation states. The inclusion of domain wall effects through the irreversible and reversible polarizations provides a model which characterizes the more gradual transitions observed in the data. The global anhysteretic for the Langevin model is similar to the Ising spin curve depicted in Figure 6a.

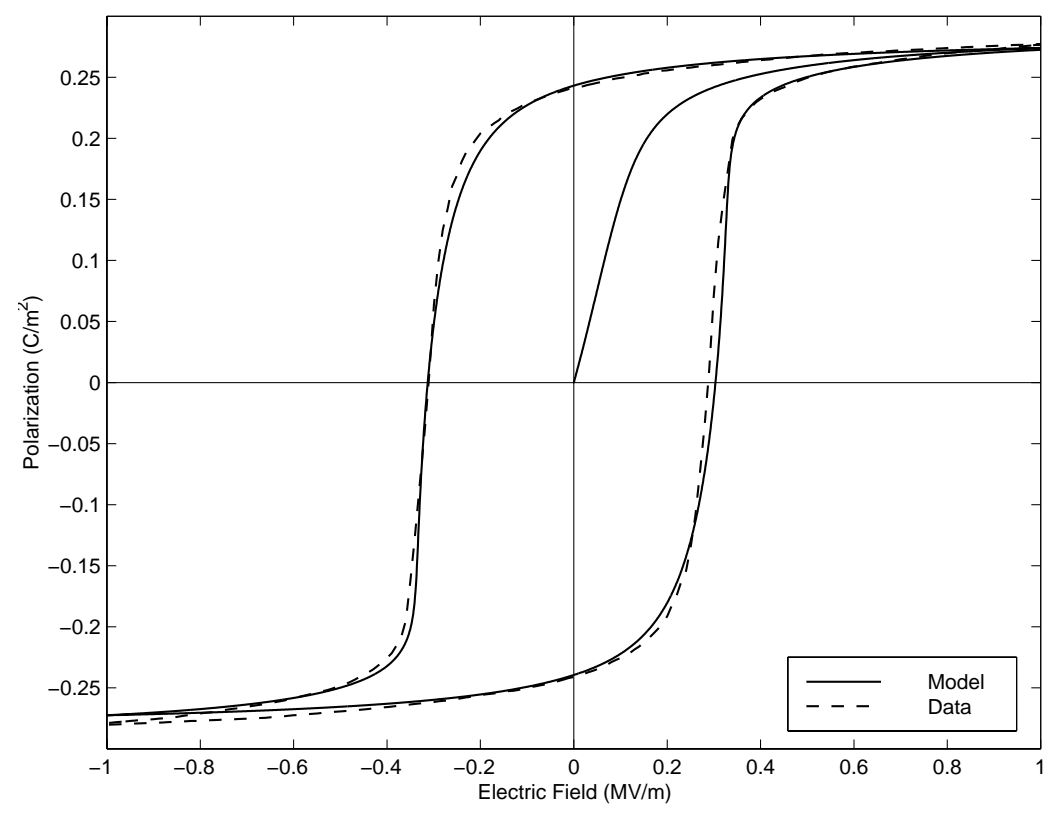

Figure 7. Experimental PMN-PT-BT data and model fit with the Langevin anhysteresis model. 


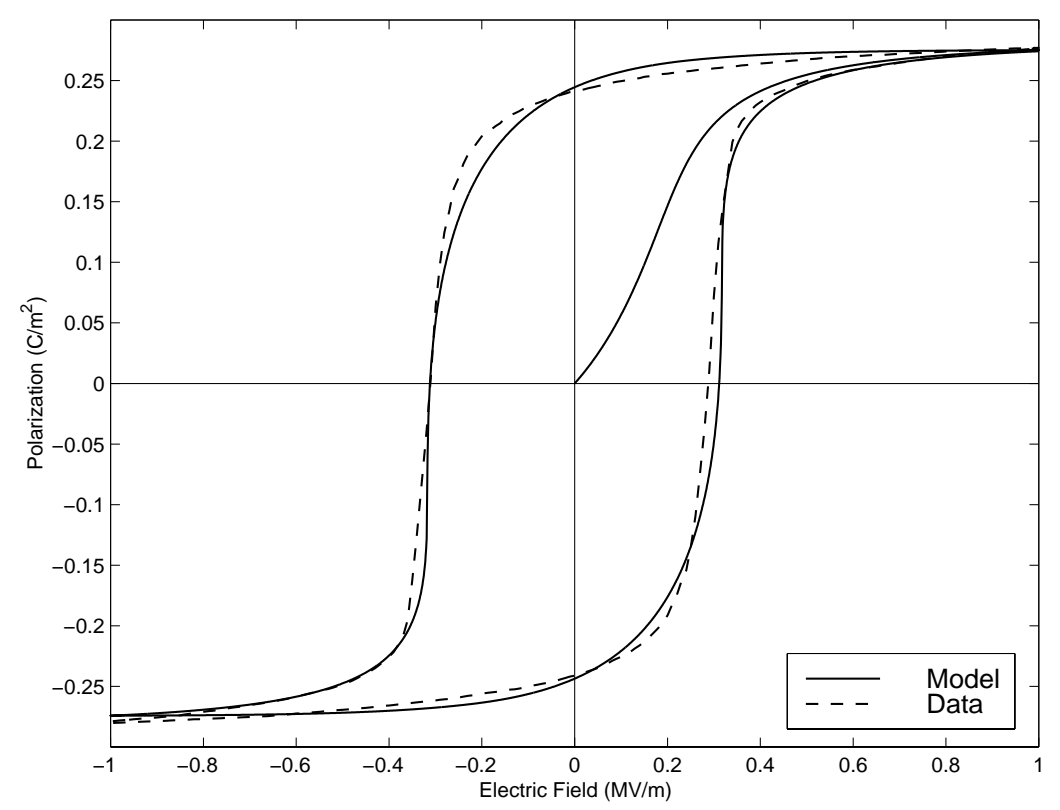

Figure 8. Experimental PMN-PT-BT data and model fit with the Ising spin anhysteresis model.

\section{Concluding Remarks}

This paper addresses the modeling of hysteresis in ferroelectric materials as well as certain relaxor ferroelectrics employed at temperatures well below the Curie point. The model is derived through the characterization of the energy required to bend and translate domain walls that are pinned at inclusions in the material. The first step in the development of the model is the determination of appropriate constitutive relations which characterize the anhysteretic $E-P$ curve. The relations quantify the global equilibrium state of the material in the idealized case that domain walls are not significantly inhibited by pinning sites in the material. Several techniques have been previously used to model anhysteretic polarization or magnetization curves including the Langevin and Ising spin relations described here. An important component of the anhysteretic model is the inclusion of interactions due to the neighboring domains through a mean field approximation. Hysteresis effects are then quantified through the computation of the average energy required to move domain walls across inclusions or pinning sites inherent to the material. The resulting quasistatic ferroelectric model is analogous to that developed by Jiles and Atherton for ferromagnetic materials.

The resulting hysteresis model is comprised of a simple differential equation whose solution provides the theoretical polarization at a given field level. The use of the model for a given application requires the determination of five parameters $\alpha, k, c, a$ and $P_{s}$ which are macroscopic averages of microscopic domain properties. Hence the model incorporates the underlying domain physics but is amenable to applications requiring real-time implementation. The determination of parameters is facilitated by the physical nature of the parameters (e.g., increased values of the average pinning energy $k$ lead to increased hysteresis) in combination with the fact that the saturation polarization can often be directly measured. Hence the model can be easily updated to accommodate the changing operating conditions often encountered in control applications.

In the form presented here, the model strictly applies to isotropic materials. Anisotropy effects are manifested primarily in the construction of the anhysteretic curve since domains will align with crystallographic easy axes rather than align in bipolar or uniform orientations. The choice of 
model for the anhysteretic polarization can be modified to accommodate specific crystallographic anisotropies if desired. In its current form, the model does incorporate symmetric minor loops. We note, however, that the present model does not accommodate certain forms of asymmetric minor loops, full electromechanical coupling, or frequency-dependent hysteresis losses. While these extensions are important for general design and control applications, they lie beyond the scope of this paper and are under investigation.

To illustrate the capabilities of the model, the characterization of data collected from a PMNPT-BT actuator at a temperature $65^{\circ}$ below the Curie temperature was considered. Both the Langevin and Ising spin relations were considered for the construction of the anhysteretic curve. The comparison of the model and experimental results illustrates that for the specified operating regime, the model provided a highly accurate characterization of the hysteresis exhibited by the material, with the Langevin-based model providing a slightly better characterization in the saturation region of the curve. While illustrated in the context of relaxor ferroelectrics at low temperatures, the electrostatic basis for the model is sufficiently general to permit its application to a variety of ferroelectric and relaxor ferroelectric materials.

\section{Acknowledgements}

The authors express sincere appreciation to David Jiles for his input regarding the development of these modeling techniques for ferromagnetic materials and Pamela Sadler-Hom for collecting the experimental data on the PMN-PT-BT actuator. The research of R.C.S. was supported in part by the Air Force Office of Scientific Research under the grant AFOSR-F49620-98-1-0180.

\section{Appendix A: Work in the Polarization Process}

The discussion in Section 3.2 provides a somewhat formal motivation for the energy functional (32). We provide here a more rigorous analysis to motivate this choice. A similar analysis for ferromagnetic materials can be found in Brown [3] with additional details regarding the magnetic model presented by Venkataraman and Krishnaprasad [52]. Note that throughout this discussion, it is assumed that field and polarization changes are sufficiently slow to permit the use of electrostatic field relations. In many applications, involving the characterization of materials, this is a reasonable assumption since experiments are conducted under quasistatic conditions.

To quantify the effective field contributions, it is advantageous to consider the work required to polarize the body rather than the work necessary to achieve a specified field level. Let $\rho=\frac{d Q}{d V}$ denote a charge density in the region $V$ as depicted in Figure 9. As detailed in [48, page 72], this charge configuration then produces the electric field

$$
\mathbf{E}=\int_{V} \frac{\rho \hat{\mathbf{r}}}{4 \pi \epsilon_{0} r^{2}} d V
$$

at the point $s$ in the dielectric material $\tau$. Note that $\hat{\mathbf{r}} \equiv \frac{\mathbf{r}}{|\mathbf{r}|}$ and $r \equiv|\mathbf{r}|$. Furthermore, the electric potential due to the dielectric is

$$
\phi=\frac{1}{4 \pi \epsilon_{0}} \int_{\tau} \frac{\mathbf{P} \cdot \hat{\mathbf{r}}}{r^{2}} d \tau
$$

(see [48, page 260]). Finally, the time rate of work due to the changing polarization is

$$
\frac{d w}{d t}=\int_{V} \rho \frac{d \phi}{d t} d V
$$


It then follows that

$$
\begin{aligned}
\frac{d w}{d t} & =\int_{V} \rho(V)\left[\frac{1}{4 \pi \epsilon_{0}} \frac{d}{d t} \int_{\tau} \frac{\mathbf{P} \cdot \hat{\mathbf{r}}}{r^{2}} d \tau\right] d V \\
& =\int_{V} \rho(V)\left[\int_{\tau} \frac{1}{\rho(V)} \frac{d \mathbf{P}}{\partial t} \cdot \frac{\rho(V) \hat{\mathbf{r}}}{4 \pi \epsilon_{0} r^{2}} d \tau\right] d V \\
& =\int_{\tau} \frac{d \mathbf{P}}{d t} \cdot\left[\int_{V} \frac{\rho(V) \hat{\mathbf{r}}}{4 \pi \epsilon_{0} r^{2}} d V\right] d \tau \\
& =\int_{\tau} \mathbf{E} \cdot \frac{d \mathbf{P}}{d t} d \tau
\end{aligned}
$$

so that

$$
\delta w=\int_{\tau} \mathbf{E} \cdot \delta \mathbf{P} d \tau
$$

The change in polarization per unit volume is then $\mathbf{E} \cdot \delta \mathbf{P}$ so the energy required to attain a given polarization level is

$$
w=\int_{0}^{\mathbf{P}} \mathbf{E} \cdot d \mathbf{P} .
$$

So far in this discussion, the field $\mathbf{E}$ has been considered to be generated by the charge configuration $\rho$. We now consider the effective field $\mathbf{E}_{e}=\mathbf{E}+\mathbf{E}_{1}$ where $\mathbf{E}_{1}=\alpha \mathbf{P}$ quantifies the field contributions due to the polarized dielectric body. Expansion of (43) yields

$$
w_{e}=w+w_{1}
$$

where

$$
w_{1}=\int_{0}^{\mathbf{P}} \mathbf{E}_{1} \cdot d \mathbf{P}
$$

denotes the electrostatic self-energy. This quantity characterizes the shape-dependent component to the energy which does not contribute to the irreversible hysteresis losses since

$$
\oint w_{1}=\oint \alpha \mathbf{P} \cdot d \mathbf{P}=0
$$

Hence we have some flexibility when specifying an appropriate energy functional which quantifies energy losses due to hysteresis.

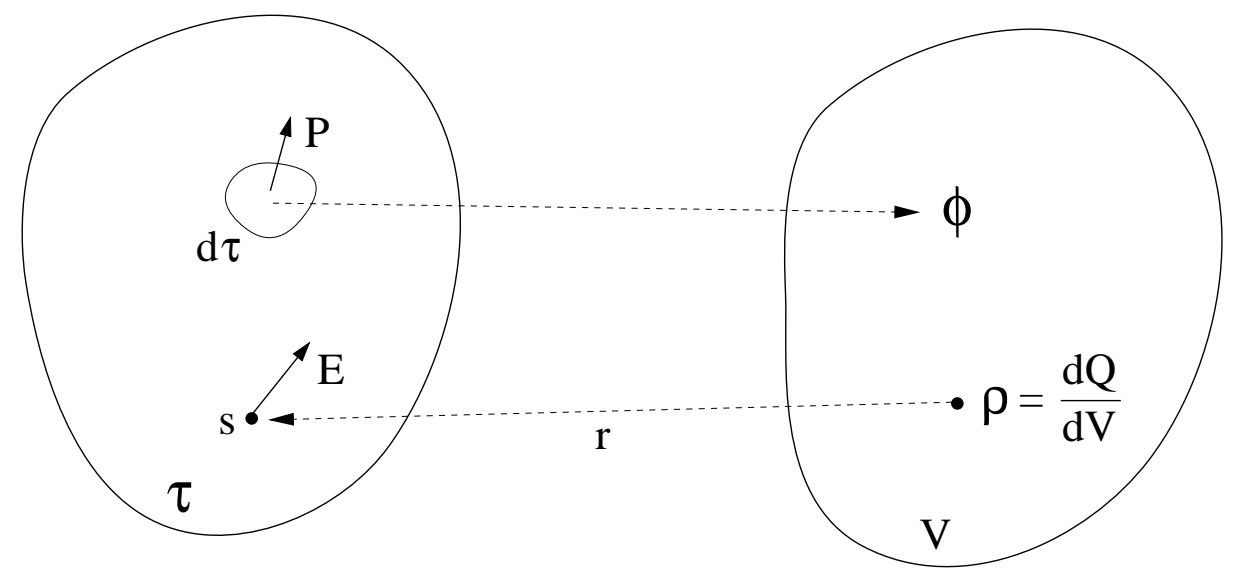

Figure 9. Orientation of the body $V$ with charge $\rho$. This generates a field $\mathbf{E}$ in the dielectric $\tau$ which in turn produces a potential $\phi$ in the charged body. 
Noting (44), the energy due to the effective field over a full polarization cycle can be expressed as

$$
\begin{aligned}
w_{e} & =\oint \mathbf{E} \cdot d \mathbf{P} \\
& =-\oint \mathbf{P} \cdot d \mathbf{E} \\
& =-\oint \mathbf{P} \cdot d \mathbf{E}-\alpha \oint \mathbf{P} \cdot d \mathbf{P} \\
& =-\frac{1}{\epsilon_{0}} \oint \mathbf{P} \cdot d \mathbf{D}_{e}
\end{aligned}
$$

for arbitrary $\alpha$. These arguments can be extended to partial cycles by employing the fact that $\int_{c_{1}} \mathbf{P} \cdot d \mathbf{P}=0$ for any closed curve $c_{1}$. This motivates the use of the functional

$$
w=-\frac{1}{\epsilon_{0}} \int_{0}^{\mathbf{D}_{e}} \mathbf{P} \cdot d \mathbf{D}_{e}
$$

when quantifying irreversible components to the polarization. We point out that (45) is identical to the functional (32), obtained through formal arguments, and is analogous to that employed by Jiles and Atherton [27] in their magnetization model.

\section{References}

[1] J.C. Anderson, Dielectrics, Reinhold Publishing Corporation, New York, 1964.

[2] H.T. Banks, A.J. Kurdila and G. Webb, "Identification of hysteresis control influence operators representing smart actuators. Part I: Formulation," Mathematical Problems in Engineering, 3, pp. 287-328, 1997.

[3] J.W.F. Brown, Magnetoelastic Interactions, Springer-Verlag, 1966.

[4] E. Callen, Y.J. Liu and J.R. Cullen, "Initial magnetization, remanence, and coercivity of the random anisotropy amorphous ferromagnet, Physical Review B, 16(1), pp. 263-270, 1977.

[5] M.E. Caspari and W.J. Merz, "The electromechanical behavior of $\mathrm{BaTiO}_{3}$ single-domain crystals," Physical Review, 80(6), pp. 1082-1089, 1950.

[6] P.J. Chen and S.T. Montgomery, "A macroscopic theory for the existence of the hysteresis and butterfly loops in ferroelectricity," Ferroelectrics, 23, pp. 199-208, 1980.

[7] I-W. Chen and Y. Wang, "A domain wall model for relaxor ferroelectrics," Ferroelectrics, 206, pp. 245-263, 1998.

[8] J-C. Debus, B. Debus and J. Coutte, "Finite element modeling of lead magnesium niobate electrostrictive materials: Static analysis," Journal of the Acoustical Society of America, 103(6), pp. 3336-3343, 1998.

[9] A.J. Dekker, Solid State Physics, Prentice-Hall, Englewood Cliffs, NJ, 1957.

[10] A.F. Devonshire, "Theory of Ferroelectrics," Advances in Physics: A Quarterly Supplement of the Philosophical Magazine, 3(10), pp. 86-130, 1954. 
[11] M.E. Drougard, "Detailed study of switching current in barium titanate," Journal of Applied Physics, 31(2), pp. 352-355, 1960.

[12] V.N. Fedosov and A.S. Sidorkin, "Quasielastic displacements of domain boundaries in ferroelectrics," Soviet Physics Solid State, 18(6), pp. 964-968, 1976.

[13] R.P. Feynman, R.B. Leighton and M. Sands, Lectures on Physics, Volume 2, Addison-Wesley Publishing Company, Reading, MA, 1964.

[14] J. Fousek and V. Janoušek, "The contribution of domain-wall oscillations to the small-signal permittivity of triglycine sulphate," Physica Status Solidi, 13, pp. 195-206, 1966.

[15] W.S. Galinaitis and R.C. Rogers, "Compensation for hysteresis using bivariate Preisach Models," SPIE Smart Structures and Materials, 1997, Mathematics and Control in Smart Structures, San Diego, CA, 1997.

[16] W.S. Galinaitis and R.C. Rogers, "Control of a hysteretic actuator using inverse hysteresis compensation," SPIE Smart Structures and Materials, 1998, Mathematics and Control in Smart Structures, San Diego, CA, 1998.

[17] P. Ge and M. Jouaneh, "Modeling hysteresis in piezoceramic actuators," Precision Engineering, 17, pp. 211-221, 1995.

[18] P. Ge and M. Jouaneh, "Tracking control of a piezoceramic actuator," IEEE Transactions on Control Systems Technology, 4(3), pp. 209-216, 1996.

[19] F. Gilletta, P. Lauginie et L. Taurel, "Relaxation diéectrique dans les cristaux de sulfate de glycocolle multidomaines," Comptes Rendus Hebdomadaires des Séances de L'Académie des Sciences, Série B, 270, pp. 94-96, 1970.

[20] M. Hayashi, "Kinetics of domain wall motion in ferroelectric switching. I. General formulation," Journal of the Physical Society of Japan, 33(3), pp. 616-628, 1972.

[21] C.L. Hom and N. Shankar, "A dynamics model for nonlinear electrostrictive actuators," IEEE Transactions on Ultrasonics, Ferroelectrics, and Frequency Control, 45(2), pp. 409-420, 1998.

[22] C.L. Hom and N. Shankar, "Modeling time-dependent behavior in relaxor ferroelectrics," Smart Structures and Materials 1998: Proceedings of the SPIE Conference on Mathematics and Control in Smart Structures, V.V. Varadan, Ed., Volume 3323, San Diego, CA, March 1-5, pp. 287-298, 1998.

[23] Y. Huo and Q. Jiang, "Modeling of domain switching in ferroelectric ceramics: an example," International Journal of Solids and Structures, 35(13), pp. 1339-1353, 1998.

[24] J. Janta, "The influence of the shape of domains on the ferroelectric hysteresis loop," Ferroelectrics, 2, pp. 299-302, 1971.

[25] D.C. Jiles, Introduction to Magnetism and Magnetic Materials, Chapman and Hall, New York, 1991.

[26] D.C. Jiles and D.L. Atherton, "Theory of the magnetisation process in ferromagnets and its application to the magnetomechanical effect," Journal of Physics D: Applied Physics, 17, pp. 1265$1281,1984$. 
[27] D.C. Jiles and D.L. Atherton, "Theory of ferromagnetic hysteresis," Journal of Magnetism and Magnetic Materials, 61, pp. 48-60, 1986.

[28] D.C. Jiles, J.B. Thoelke and M.K. Devine, "Numerical determination of hysteresis parameters for the modeling of magnetic properties using the theory of ferromagnetic hysteresis," IEEE Transactions on Magnetics, 28(1), pp. 27-35, 1992.

[29] C. Kittel, Introduction to Solid State Physics, John Wiley and Sons, New York, 1976.

[30] B.D. Laikhtman, "Flexural vibrations of domain walls and dielectric dispersion of ferroelectrics," Soviet Physics Solid State, 15(1), pp. 62-68, 1973.

[31] R. Landauer, D.R. Young and M.E. Drougard, "Polarization reversal in the barium titanate hysteresis loop," Journal of Applied Physics, 27(7), pp. 752-758, 1956.

[32] E.A. Little, "Dynamic behavior of domain walls in barium titanate," Physical Review, 98(4), pp. 978-984, 1955.

[33] K. Mayergoyz, Mathematical Models of Hysteresis, Springer-Verlag, New York, 1991.

[34] W.J. Merz, "Domain formation and domain wall motions in ferroelectric $\mathrm{BaTiO}_{3}$ single crystals," Physical Review, 95(3), pp. 690-698, 1954.

[35] R.C. Miller, "Some experiments on the motion of $180^{\circ}$ domain walls in $\mathrm{BaTiO}_{3}$," Physical Review, 111(3), pp. 736-739, 1958.

[36] R.C. Miller and A. Savage, "Motion of $180^{\circ}$ domain walls in metal electroded barium titanate crystals as a function of electric field and sample thickness," Journal of Applied Physics, 31(4), pp. 662-669, 1960.

[37] R.C. Miller and G. Weinreich, "Mechanism for the sideways motion of $180^{\circ}$ domain walls in barium titanate," Physical Review, 117(6), pp. 1460-1466, 1960.

[38] S.L. Miller, R.D. Nasby, J.R. Schwank, M.S. Rodgers and P.V. Dressendorfer, "Device modeling of ferroelectric capacitors," Journal of Applied Physics, 68(12), pp. 6463-6471, 1990.

[39] T. Mitsui, I. Tatsuzaki and E. Nakamura, An Introduction to the Physics of Ferroelectrics, Gordon and Breach Science Publishers, New York, 1976.

[40] M.L. Mulvihill, L.E. Cross and K. Uchino, "Low-temperature observation of relaxor ferroelectric domains in lead zinc niobate," Journal of the American Ceramic Society, 78(12), pp. 3345-3351, 1995.

[41] S. Nomura, M. Endo and F. Kojima, "Ferroelectric domains and polarization reversal in $\mathrm{Pb}\left(\mathrm{Zn}_{1 / 3} \mathrm{Nb}_{2 / 3}\right) \mathrm{O}_{3}$ crystal," Japanese Journal of Applied Physics, 13(12), pp. 2004-2008, 1974.

[42] M. Omura, H. Adachi and Y. Ishibashi, "Simulations of ferroelectric characteristics using a one-dimensional lattice model," Japanese Journal of Applied Physics, 30(9B), pp. 2384-2387, 1991.

[43] N.A. Pertsev and A.G. Zembil'gotov, "Microscopic mechanism of polarization switching in polymer ferroelectrics," Soviet Physics Solid State, 33(1), pp. 165-171, 1991. 
[44] J.C. Piquette and S.E. Forsythe, "A nonlinear material model of lead magnesium niobate (PMN)," Journal of the Acoustical Society of America, 101(1), pp. 289-296, 1997.

[45] C.F. Pulvari and W. Kuebler, "Phenomenological theory of polarization reversal in $\mathrm{BaTiO}_{3}$ single crystals," Journal of Applied Physics, 29(9), pp. 1315-1321, 1958.

[46] E.M. Pugh and E.W. Pugh, Principles of Electricity and Magnetism, Addison-Wesley, Reading MA, 1970.

[47] M.J. Sablik and D.C. Jiles, "Coupled magnetoelastic theory of magnetic and magnetostrictive hysteresis," IEEE Transactions on Magnetics, 29(3), pp. 2113-2123, 1993.

[48] A. Shadowitz, The Electromagnetic Field, Dover Publications, New York, 1975.

[49] R.C. Smith, "Inverse compensation for hysteresis in magnetostrictive transducers," CRSC Technical Report CRSC-TR98-36; Mathematical and Computer Modeling, submitted.

[50] G.A. Smolenskii, V.A. Bokov, V.A. Isupov, N.N. Krainik, R.E. Pasynkov and A.I. Sokolov, Ferroelectrics and Related Materials, G.A. Smolenskii, Editor in Chief, Translated from the Russian by S.J. Amoretty, Gordon and Breach Science Publishers, New York, 1984.

[51] J.A. Tuszyński, B. Mróz, H. Kiefte and M.J. Cloutier, "Comments on the hysteresis loop in ferroelastic $\mathrm{LiCsSO}_{4}, "$ Ferroelectrics, 77, pp. 111-120, 1988.

[52] R. Venkataraman and P.S. Krishnaprasad, "Qualitative analysis of a bulk ferromagnetic hysteresis model," Proceedings of the IEEE Conference on Decision and Control, Tampa, FL, December 16-18, 1998, to appear.

[53] I.K. Yoo and S.B. Desu, "Modeling of hysteresis in ferroelectric thin films," Philosophical Magazine B, 69(3), pp. 461-469, 1994.

[54] X.D. Zhang and C.A. Rogers, "A macroscopic phenomenological formulation for coupled electromechanical effects in piezoelectricity," Journal of Intelligent Material Systems and Structures, 4, pp. 307-316, 1993. 